\title{
Deaf People with Wardenberg Syndrome Type II: Jordanian Case Study
}

\author{
Dr. Samer Mohammad Abu Drei \\ Imam Abdulrahman Bin Faisal University, \\ Saudi Arabia
}

DOI: https://doi.org/10.36941/jesr-2020-0o78

\section{Abstract}

This study aimed to identify deaf people with Deaf with Waardenburg Syndrom Type II. In order to achieve the objectives of the study, the qualitative method was used for the one case study. The sample included (2) deaf students from all deaf schools in Jordan, who are between the ages of (9-10), and (Wechsler Intelligence Scale ,WISC-IV) was applied in the language of Performance Scales and Audiometry. The results of the study showed the presence of symptoms in Deaf people with Wardenberg syndrome type II in terms of: auditory sensory loss. The presence of a side shift to the inner corner of the eyes. Premature graying of hair on the front of the head in white and some on the eyelashes or on the eyebrow. The eyebrows are connected to each other. Dark blue or brown eyes with black and pigment color differences in the iris. The root of the nose is wide and the presence of the rabbit's lip. The results of the study also showed that there is an intense focus during communication in the sign language at the hands of the speaker due to the fact that the color contrast of the eyes led to a dispersion of the focus in the sign language. They also have a sense of the direction of light, which may adversely affect the process of visual communication of sign language. The results of the study also showed that the average hearing impairment was the highest category. The study recommends further studies and research related to the level of intelligence of deaf people with Type II syndrome, as well as studies related to visual perception in Deaf people with type 2 Wardenburg syndrome and the inclusion of this syndrome as an important part of the categories of hearing impairment.

Keywords: Deaf, Wardenberg syndrome, Hearing impairment syndromes

\section{Introduction}

Waardenburg syndrome, WS syndrome is a rare and unusual disease characterized by sensory nerve deafness, pigmented deformities as well as defects of auditory nerves. With additional symptoms, Wardenburg syndrome is classified into four types (WS1, WS2, $\mathrm{WS}_{3}$, and $\left.\mathrm{WS}_{4}\right)$, which are called:

1. Waardenburg Syndrome Type 1, WS1.

2. Waardenburg Syndrome Type 2, WS2.

3. Wardenburg syndrome of the third type, also called Klein-Waardenburg syndrome, (Waardenburg Syndrome Type 3, WS2).

4. Type 4 syndrome, also called Waardenburg-Shah, Waardenburg Syndrome Type 4, WS4.

This specialized research for Wardenburg syndrome is the second type for people with hearing disability from rare Arab research (according to the researcher's knowledge) and this in turn confirms that the category of hearing disability is a heterogeneous group, according to the degree of auditory loss, and the syndrome is considered a non-infectious disease, and it can't be treated with drugs And it cannot happen due to lifestyle or growth factors, as many books specializing with hearing 
impairment and theoretical literature that are almost identical have not indicated and they were not educationally discovered, which led to the emergence of a new pediatric syndrome known as Wardenburg syndrome within the class. Deaf, if any, the information provided is almost insufficient to know the truth of this syndrome. Therefore, the measurement and diagnosis of Wardenburg syndrome for people with hearing disability is one of the issues whose folds have aspects of great difficulty, whether it is medical (for the cost of examining the gene that causes the syndrome) in general or psychometric (because there are no specialized measures for people with auditory disability of Wardenburg syndrome whether it is for mental ability, perception, Sign Language) or pedagogical standards that are appropriate to their ability to use sign language.

Hence the current study came to provide an explanation of the characteristics of deaf people with Type II syndrome by knowing the symptoms and sign language for them.

\section{Literature Review}

In (1947), the disease was discovered by the Dutch ophthalmologist Dr. Petrus Johannes Waardenburg, where he observed that the color of the eyes of some of those who had designs differed from each other. Although the prevalence of this disease reaches one case per (20000-400oo) newborn children, it affects approximately $(3 \%)$ of the total of those who have been designed since birth and their characteristics were as follows:

- Congenital hearing loss.

- The presence of a side shift to the inner corner of the eyes.

- Variation in skin pigmentation (albinism).

- White premature graying of hair at the front of the head.

- The eyebrows are bound together (excess eyebrows sticking together in the midline).

- Blue eyes and pigment color differences in the iris (Heterochromia Iridium).

- The root of the nose is wide. (Waardenburg, 1951).

Until this syndrome became known as Waardenburg Syndrome Type 1, WS1.

Then Arias (1971) defined Wardenburg syndrome of the second type (WS2). As individuals of the second type ( $\mathrm{WS}_{2}$ ) have all the characteristics of the first type (WS1) except for the angle of the eye. (Pantke, Cohen, 1971).

The classifications of this syndrome have also evolved with different symptoms, and therefore we can differentiate between those subtypes of Wardenburg syndrome and work on knowing ways of communication that are appropriate to the type and severity of the problem.

Wardenburg syndrome is considered to be a heterogeneous syndrome. Doctors have identified four types with different symptoms. Individuals with this syndrome have symptoms similar to each type, but type I and II are the most common for this syndrome:

1. Wardenburg syndrome type 1 : This syndrome is characterized by the fact that the individual has a large distance between his eyes. Where $(20 \%)$ of them suffer from hearing loss, especially the inner ear. They have a difference in the color of the iris or the eyes together, and the appearance of white hair on his head, whether it is at the front of the head or a dispenser. (Hart, Miriyala, 2017)

2. Wardenburg syndrome type two: Hearing loss is more common in this type compared to type I, and their hearing loss is $(50 \%)$. Symptoms are similar to type I, including changes in hair, skin, and eyes. This type is distinguished from the first type mainly because there is not much distance between the eyes. (Jalilian et al, 2018)

3. Wardenburg syndrome type III: The third type is similar to the first and second types, and often results in loss of hearing and a change in the pigment of the iris of the eyes. People who own this model usually have a great distance between their eyes and their wide nose. The distinguishing feature of this type that distinguishes it from the first and the second type is the presence of problems with the upper limbs, as those with the third type have problems and deformities in the arm or shoulders or deformities of the joints. Some also 
suffer from mental disabilities or the rabbit's lip accompanying this type. The third type is also called Klin-Wardenburg syndrome. (Klein, 1983)

4. Wardenburg syndrome, type IV: Type IV causes changes in the pigment of the iris and may also lead to hearing loss. People with this type of disease have a colon disorder, which causes severe constipation. (Shah et al., 1981).

The National Institute of Deafness and Other Communication Disorders (NIDCD, 2007) defines Wardenburg syndrome as a rare genetic disorder characterized by hearing and pigmentation mutations.

He also indicated (Pingault et al, 2010) that Wardenburg syndrome is the leading cause of symptomatic sensorineural hearing loss (SNHL) and is a disease that causes visual disturbance, eyebrows, iris divergence, and white hair.

Wardenburg (1951) notes 6 main characteristics related to Wardenburg syndrome types:

Wide nasal root, partial or total pigmentation differences in the iris of the eye, from birth, brow attachment, and white hair to the front of the head (Tajra et al., 2006).

Arfanet (Orphanet, 2018) considers the Wardenburg syndrome a hearing disorder that affects between $(2-3 \%)$ congenital deafness. Estimated cases around the world are ( 2 to 3 cases per 100,0oo) of society, and equally affect both sexes.

(Nayak, Chetan S; Isaacson, Glenn, 2003) indicate that the incidence of Wardenburg syndrome has been reported in societies, indicating that the clinical characteristics of two categories: one of European origin and the other of South Asia. Where the report indicated that Wardenburg syndrome is between $(2-5 \%)$ of cases of congenital deafness. It was first described in the northern European regions, particularly for those with white skin.

(Harlor, Bower, 2009) confirms that Wardenburg syndrome is a high sign of hearing loss. Even if newborns are examined, and the American Academy of Pediatrics recommends referring at least one diagnostic evaluation, These children are also evaluated no later than every (3) months of age. Hearing loss requires appropriate referral through (otolaryngology, audiology, genetics) and intervention no later than (6) months of age.

Wardenburg syndrome usually has distinct changes in hair and skin, as well as sensory nerve hearing loss. In this minor variation, symptoms may be hidden and may not be diagnosed until either a family member is diagnosed or all family members are examined. (Egbalian, 2008).

Deaf people with this syndrome have eye changes such as each eye in a different color from the other, or hair or skin, which can be seen easily. The degree of hearing loss is not always constant but varies from simple to severe hearing impairment and may be mono or bilateral. (Toriello et al, 2004).

The American National Institute of Medicine (USNLM, 2006) indicates that not everyone with Wardenburg has a hearing loss. It usually affects only hearing impaired (6o\%) of those diagnosed with the syndrome.

In (2009) (Adameyko et al, 2009) presented three sets of evidence on a large number of pigment cells and how they affect the skin. Dermatological analysis (congenital birthmarks) has led to the emergence of a new theory proposing the existence of a new group of primary cells derived from melanocytes originating in the mesoderm, which arises due to centrifugal migration.

The melanocytes are almost complex, and are responsible for the color difference of the skin, eye (iris), skin, and cochlea (Guerlin, 2010; Jones, 2006).

Steel et al (1989) It also confirms that Wardenburg syndrome affects physical features due to the absence of melanocytes, especially in the inner ear (cochlea) through the vascular layer, that the hearing will be affected.

(Hager et al, 2010) also indicates that Wardenburg syndrome is a rare disease characterized by loss of sensory nervous system and chromosomal abnormalities of the iris, skin and hair due to mutations in the gene $\left(\mathrm{PAX}_{3}\right)$.

In most individuals, Wardenburg syndrome ( $\left.\mathrm{WS}_{1}\right)$ is diagnosed by performing a physical 
examination to search for clinical criteria that include:

Loss of sensory hearing.

Pigment changes in hair and eyes. (Shields et al, 2013)

This syndrome can be recognized by calculating the Wardenburg index (W), which are the necessary measurements for the calculation of the $\mathrm{W}(\mathrm{mm})$ index. In the case of suspicion of Wardenburg syndrome, an appropriate diagnostic evaluation may include the use of a caliper (used to measure thickness or diameter), This syndrome can be diagnosed by measuring the distances between the inner corners of the eye and the outer corners of the eye, by means of a (W) indicator to check whether or not the angle of the eye (Dystopia canthorum) is present. which is the result that It may suggest that we have Wardenburg syndrome type I J is as follows:

1. The distance to the inner eye angle (a).

2. The distance between the two pupils (b).

3. The outer distance of the angle of the eye (c).

It is through the following formula:

$\mathrm{X}=(2 \mathrm{a}-(0.2119 \mathrm{c}+3.909)) / \mathrm{c}$

$\mathrm{Y}=(2 \mathrm{a}-(0.2479 \mathrm{~b}+3.909)) / \mathrm{b}$

$\mathrm{W}=\mathrm{X}+\mathrm{Y}+\mathrm{a} / \mathrm{b}$

Mathematical results are an eye angle greater than ( $\mathrm{W}<1.95$ index).

Through this equation, the diagnosis of Wardenburg syndrome can be measured from birth or early childhood through comprehensive clinical assessment and determination of results and their physical characteristics. (Farrer et al, 1992)

The specific features of the face that were mentioned previously.

When talking about the relationship between Wardenburg syndrome and mental disability, there is a difference about the mental ability of Deaf people with Wardenburg syndrome.

Another report (Pasteris et, 1992) of a case study of Wardenburg syndrome suggests that there are symptoms including:

- Small size of the head.

- Having a mental disability.

- Severe physical disorders and abnormalities.

(Melville \& Cameron, 2003) also confirm that Deaf people (before language acquisition) have Wardenburg syndrome that this relationship has not been reported in theoretical literature. We believe that there is astonishing similarity to these two people, which gives us the possibility of the presence of apparent behavioral patterns by describing the cases in the presence of:

- Fragile Syndrome (X).

- German measles.

- Herpes simplex encephalitis.

The second type interferes with the first type of syndrome in terms of characteristics, which is also easy to identify, especially when the reader or researcher in the field of deaf knows some of its symptoms, but do not forget that the category of deaf or hard of hearing people with Wardenburg syndrome the second type can also be discovered through schools Specialized for deaf people due to hearing problems.

The researcher should search for a set of symptoms or features that distinguish the second type of syndrome from others, which include the following:

- Iris color variation (contrast) in the right eye (47\%).

- Decreased pigmentation of the iris of the left eye.

- Eyebrows coupling.

- The presence of white hair at the front of the head.

- Heterogeneous class.

- Sensory hearing loss (77\%).

It indicates that the occurrence of Wardenburg syndrome has been reported in societies, indicating that the clinical characteristics of two categories: one of European descent and the other 
area of South Asia. The report indicated that this syndrome ranges between $(2 \%-5 \%)$ of congenital deafness. It was first described in northern European regions, especially for those with white skin. (Nayak, Chetan S; Isaacson, Glenn, 2003).

Through research by (Dourmishev et al, 1999) four types of Wardenburg syndrome were identified. As these types are known to be more common through Type I (WS1) and Type II (WS2), which are characterized by the presence of severe hearing impairment and the prevalence of hearing impairment is higher in deafness of the type II ( $\mathrm{WS}_{2}$ ) more than the first type (WS1). Deaf and hardof-hearing individuals may also experience other birth defects, such as the rabbit's lip. Wardenburg's type II syndrome is often called narrow-angle, but uncommon, eye-shaped Wardenburg syndrome.

(Nayak, Isaacson, 2003) believes that hearing loss is not universally linked to Wardenburg syndrome, but it was noted that there were problems with nervous hearing loss, that (69\%) had Wardenburg syndrome type I and (87\%) had Wardenburg syndrome type II. The defects are caused by a change in the pigmentation of the skin (8.3\%) of the patients.

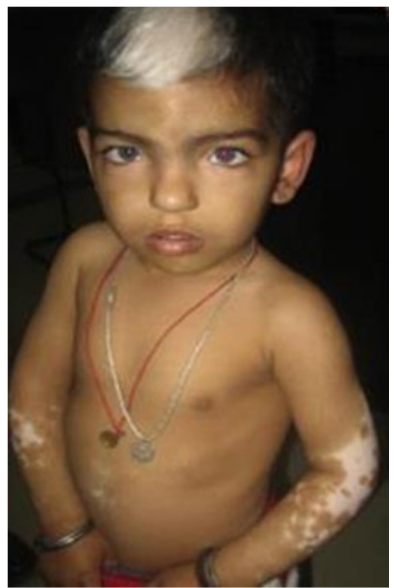

Associated skin diseases (Vichare, Bhargava, 2013)

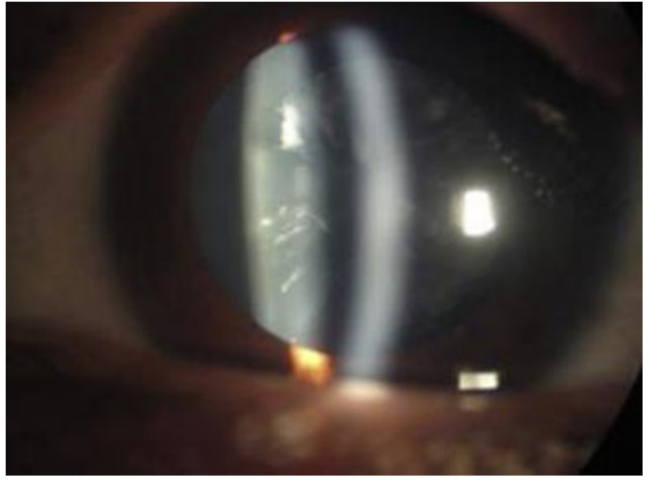

White water in the eye of Wardenburg syndrome is considered a rare case (Vichare, Bhargava, 2013)

Thus, Wardenburg syndrome is considered a rare disease, afflicting approximately ( 1 in 40,0oo people), that is, approximately ( 2 to $5 \%$ ) of all deafness caused by gene abnormalities, which is the result of the syndrome. Despite its rarity, Wardenburg syndrome may be common in a family because it is hereditary.

(Dourmishev, 2013) confirms the second type of Wardenburg syndrome caused by mutations in genes (MITF and SNAI2). Sensory hearing loss (77\%) and iris color variation (47\%) are the most important diagnostic indicators for this type.

(USNLM, 2006) asserts that type (II) is linked to a mutation in MITF and SNAI2. It also indicates (Milunsky, 2007) that about (10-20\%) of patients with Type II syndrome were caused by the emergence of a mutation in a gene (MITF).

Although there are no Arab studies (according to the researcher's knowledge) that confirm the understanding and characteristics of deaf people with Wardenburg syndrome of the second type, those cases were discovered when Abu Drei conducted an extensive study in (2017) entitled "Standardization A Jordanian Version of The Wechsler Intelligence Scale " for Children -Fourth Edition Adapted for Deaf Via Sign Language From $(6-16.11)$ years " This study aimed to legalize the Jordanian image of the (wisc-4) at school stage for the age group (6 - 16.11) years to measure intelligence in sign language for the deaf. In order to achieve the objectives of the study, the wisc-4 IQs were converted after indications of their validity, reliability, and criteria of the wisc-4 scale in the 
ordinary and deaf samples were converted into sign language. The sample of the study included the deaf and ordinary (831), and indications of the validity of the Jordanian image from the (wisc-4) scale in the sign language of the deaf were represented in the honesty of the content, discriminatory honesty, sincerity of the building, and associative honesty, as well as indications of consistency The scale was the use of Alpha Cronbach, the method of return, and the residents' agreement.

It was also reached to the criteria of the scale represented by converting the raw grades to standard degrees and then to an IQ. The results of the study showed:

- There were statistically significant differences $(\alpha=0.05)$ in performance on the sub-tests of the Jordanian image of the scale (wisc-4) of children's intelligence in the school stage due to a variable in the ordinary and deaf category and the gender variable in the deaf group.

- There were statistically significant differences $(\alpha=0.05)$ in performance on the sub-tests of the Jordanian image of the scale (wisc-4) for deaf children in the language of sign at school due to the change in the degree of hearing disability (simple - medium - severe - cochlear simple + cochlear) for the category Age (6-16.11) years.

- There are statistically significant differences in performance on the subtests of the Jordanian image of the scale (wisc-4) of children in school due to the age variable for the deaf in the sign language in favor of the age group (13.00-13.12).

(Jian, Frederic, Yong, Paul, Kris, Ingeborg M, 2015) conducted a study entitled "Hearing loss in Wardenburg syndrome: a literature review" aimed at identifying auditory and genetic problems of the syndrome. Calculate the prevalence rates of auditory loss associated with different types and genes of Wardenburg syndrome. A sample included (73) articles, and (417) patients were described. The results showed that:

- Auditory loss was (71\%) and was significantly associated with Wardenburg syndrome.

(Jalilian et al.,2018) conducted a study titled "Comprehensive Genetic and Clinical Assessment of Type II Wardenburg Syndrome among an Iranian Sample of Patients" aimed at identifying the clinical and genetic characteristics of type II syndrome. The study sample included (31) Wardenburg syndrome type II, (22) men and (9) women, the patients underwent a genetic mutation examination (MITF, SOX10, SNAI2). The results showed the following:

- Variation in prominent manifestations of Wardenburg syndrome, type II.

- The presence of auditory sensory hearing disability.

- The presence (variation) of iris is the most common (67\% and $45 \%)$, respectively.

- The presence of a loss of sense of smell is considered one of the rare and less common cases.

The presence of auditory sensory impairment, which in turn affects the deaf people with Wardenburg syndrome (Liu, Newton, Read, 1995) conducted a study entitled "Auditory loss and chromosomal disorders in Wardenburg syndrome with reference to the second type of syndrome" This study aimed to know The impact of Wardenburg syndrome, the study sample included (30) families, where it was found that (149) people were affected by Wardenburg syndrome, causing hearing loss and chromosomal abnormalities. Where (22) families included (89) people who were affected by the second type of Wardenburg syndrome and (8) families who had (6o) people affected by the first type of Wardenburg syndrome, where they found bilateral sensory hearing loss to be the most common degree of hearing loss was (100 dB) with no differences between the types of syndrome. Although there was no significant difference in pigmentation disorders between the first and second types, the iris color change was more common in the second type of the first type and the Pigmentation disorders of the first type were more common (62\%), And the first type is more than one defect, but only $(28 \%)$ of the second type, the chromosomal disturbances were not noticeable in the group of hearing impairment than in the listeners group usually in the first or second type. There was a significantly higher proportion of chromosomal defects in the hearing impairment group in the first type. (93.3\%) of type I patients and (88.5\%) Among patients with type 2 hearing impairment, the recurrence of pigmentation disorders was not greater when hearing loss was more severe in both types. Hearing loss and chromosomal abnormalities were a marked contrast.

Karan and Archana (2015) conducted a study entitled "Wardenburg syndrome: a case study of 
two patients". This study aimed to know the characteristics of this syndrome in India, where the sample included (2) of the brothers who are (12) years old and they have a hearing loss with both ears and have symptoms with Wardenburg syndrome. Like a distinctive white hair in the midline with a stunning two-blue iris color. There was also slight white pigmentation on the right forearm. There was also slight white pigmentation on the right forearm. As well as pigmentation of the eyes. The second case in this study was her younger brother, who had two blue eyes, and a white forehead of hair. Their father had a history of graying hair. The results showed:

That the first patient has the following advantages of Wardenburg syndrome, including:

- Hypersensory hearing impairment.

- A prominent white forehead of hair.

- Double Blue Iris.

- White pigmentation on the arm.

- Family history of this disease.

- The younger brother has a blue iris and a white facade of hair.

As for the second patient in this study, he has the following advantages in line with Wardenburg syndrome, including:

- A white introduction to the hair has been present at birth.

- Double Blue Iris.

- White pigmentation on the arm.
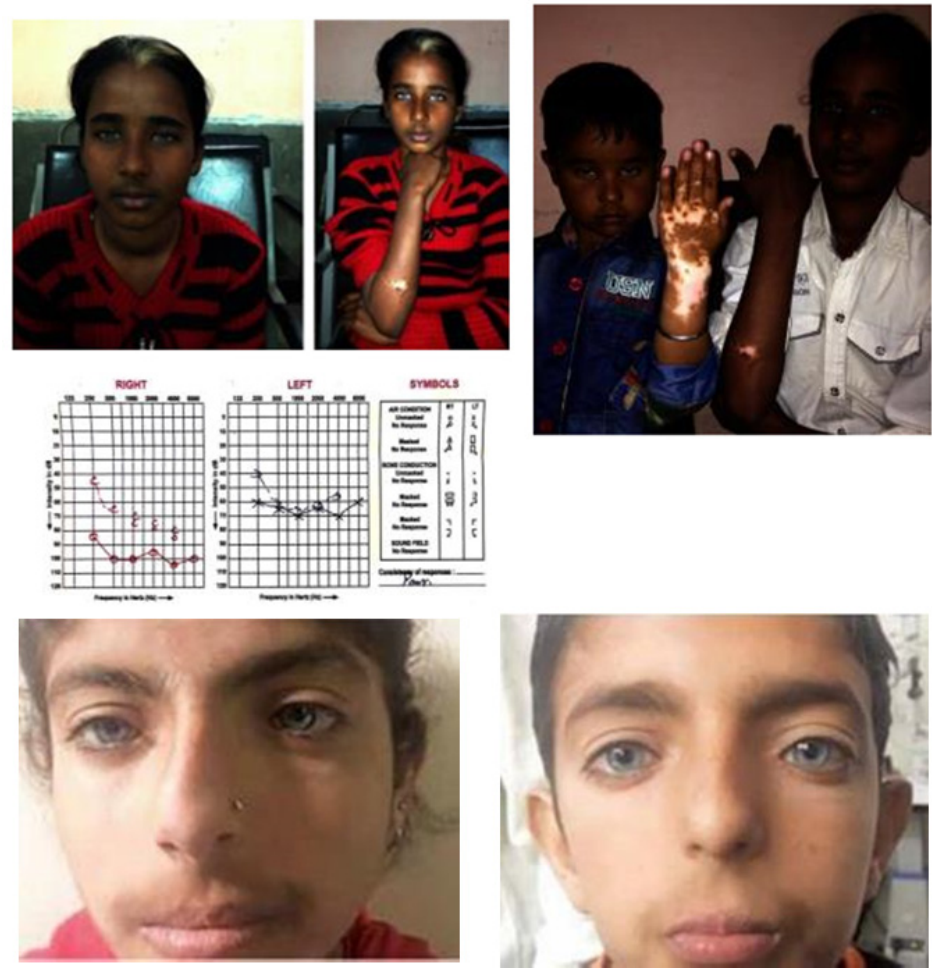

(Kritika, Indu, Gaurav, 2019) conducted a study titled "A case study of two siblings from Wardenburg syndrome." This study aimed to identify the classic features of Wardenburg syndrome among siblings. The sample included (2) siblings as the female (18) a year and her younger brother at the age of (14) years. Where the female had blue eyes, and the presence of bonding with the eyebrows and the 
presence of a wide distance between the corners of the eye, as well as the front of the hair was white with a gray color, and hypopigmented spots in her hands and feet and the nose root She was also suffering from severe hearing loss of (98.3) decibels in the right ear and (96.6) dis It was in the left ear. Her IQ was normal and the MRI was normal. The eye examination was $20 / 20$ vision in both eyes. Her younger brother had severe hearing loss of (96.6) decibels in the right ear and (98.3) decibels in The left ear with the root of the nose was wide. He had congenital anomalies in his right thumb. His IQ was also normal and his brain was MRI normal. The eyes examination was 20/20 vision in both eyes. He had iridescent blue in pigmentation. The pressure inside the eye was normal.

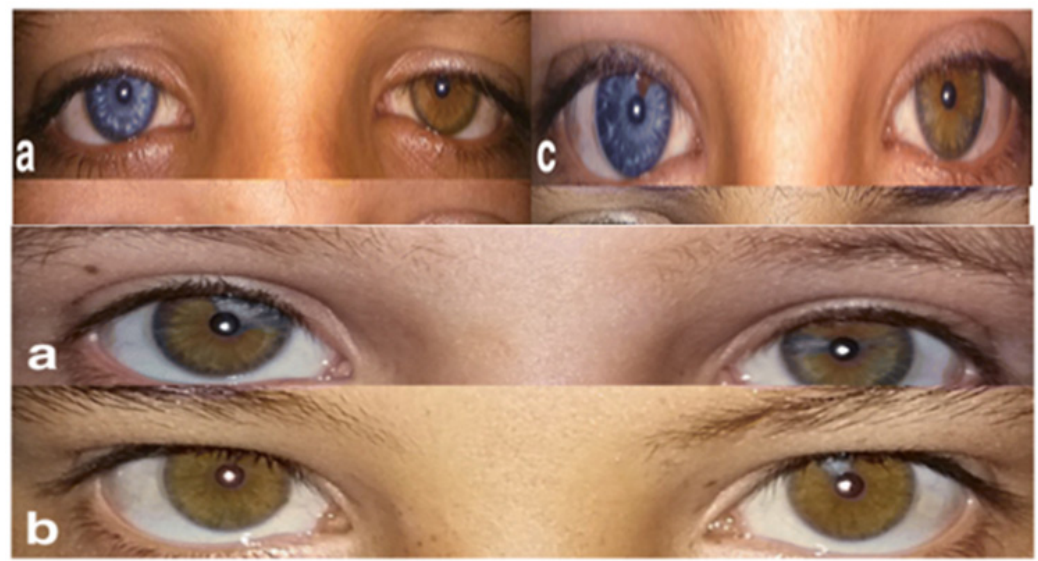

(Luma, Mohamed, Majd, 2018) also conducted a study entitled "A rare case of seven brothers suffering from Wardenburg syndrome: a case report". The study aimed to identify Wardenburg syndrome as a group of rare genetic conditions. The case was presented to a Syrian family, consisting of (14) members of the family with a low income, who came to our institute with their (8) year old son who had congenital hearing impairment. He also (6) of the brothers had a congenital auditory loss, as they were examined through early auditory brain response tests .Acoustic and audio emissions were tested and showed sensory hearing loss. One of the interesting features of the other seven brothers and sisters is that some of them have heterochromatic insufficiency, while others have segmental eye color contrast (part of the eye). Dermatological, laboratory, and x-ray consultations on the chest were examined for all brothers, and no abnormalities were detected. They had no musculoskeletal system or intestinal disturbances. Depending on the Wardenburg criteria, the brothers have Wardenburg syndrome. The results showed that although inheritance of Wardenburg syndrome is the dominant physically, we are reporting a unique condition of seven brothers with Wardenburg syndrome. The case report explains this crucial role for parents and the degree of their relationship that influenced this syndrome and which indicates that the number of children with this rare disorder is increasing.

\section{Research Questions}

The study answers the following questions:

1. What is the concept of Deaf people with Wardenburg syndrome type II on a Jordanian sample?

2. What are the characteristics of Deaf people with Wardenburg syndrome type II on a Jordanian sample?

3. What is the level of hearing impairment for Deaf people with Wardenburg syndrome type II on a Jordanian sample? 


\section{Study Significant}

Knowledge of Deaf people with Wardenberg syndrome type II was provided on a Jordanian sample.

First: theoretical importance:

1. Arouse interest in the knowledge of Wardenburg syndrome for people with hearing impairment.

2. Knowing the relationship between the degree of auditory loss and Wardenburg syndrome of the second type.

3. The scarcity of Jordanian studies that deal with Deaf people with Type II syndrome.

4. Enriching the Arabic library with a category related to hearing disability.

Second: The practical importance:

1. Determining the characteristics of Deaf people with Wardenburg syndrome type II.

2. Helping researchers benefit from the results of this study in dealing and communicating with Deaf people with Wardenburg syndrome and determining their mental ability.

\section{Study limitations}

This study has some limitations:

1. The scarcity of Jordanian studies that deal with deaf people with Type II syndrome.

2. The scarcity of studies dealing with sign language with Deaf people with Wardenburg II syndrome.

\section{Definitions of Terms}

1. Deaf : They are individuals who use sign language, alphabet, lip language, and the total way to communicate with each other, whether with or without the use of auditory aids, and their hearing aids range from $(25$ - 90) decibels.

2. Wardenburg syndrome, the second type : it is a group of rare features that appear on the individual that distinguishes them from others, which include the expansion of the root of the nose, which causes expansion of the eyes, and the appearance of a difference in the iris color of both eyes, which makes the shape of the face unusual and the presence of other changes in their appearance that are White hair (gray hair) in early childhood until before (30) years, as well as auditory loss, and the presence of some developmental problems, and this type is distinguished from the first type mainly by the lack of a large distance between the eyes.

\section{Methodology: Method and Procedures}

\subsection{Method of study}

A qualitative approach was used to suit the purposes of the current study, which is based on the evidence of the interview and observation, in addition to relying on the (coding) test from the Jordanian image of the WISC-4 scale for deaf intelligence in the sign language to determine the level of visual problems among study members.

\subsection{The sample of study}

Qualitative studies depend on a limited number of respondents, whereby the study members were identified through the researcher's follow-up of deaf cases from a community of (947) deaf students, and the researcher working in the field of hearing disability diagnosis Their number $(n=2)$ is singular within the age group (9-10 years), who were chosen intentionally from the total community of the 
deaf, and registered in schools of education.

Repeats and percentages were used to describe the study sample members

Table (1): Distribution of study sample individuals according to age

\begin{tabular}{|c|c|c|}
\hline percentage & Repetition & Age \\
\hline 50 & 1 & 9 \\
\hline 50 & 1 & 10 \\
\hline 100.0 & 2 & Total \\
\hline
\end{tabular}

Table (2): Distribution of study sample individuals according to gender

\begin{tabular}{|c|c|c|}
\hline percentage & Repetition & gender \\
\hline 50 & 1 & Male \\
\hline 50 & 1 & female \\
\hline 100.0 & 2 & Total \\
\hline
\end{tabular}

7.3 The study tool

1. Case study questionnaire (syndrome symptoms): A list of symptoms for this syndrome was prepared through theoretical framework and previous studies of the syndrome, which include:

- Congenital hearing loss.

- The presence of a side shift to the inner corner of the eyes.

- Variation in skin pigmentation.

- White gray hair on the front of the head.

- The eyebrows are bound together.

- Blue eyes or pigment color differences in the iris.

- The root of the nose is wide.

2. Auditory examination.

\subsection{Research procedures}

To achieve the aims of the study, the following measures were taken:

1. Syndrome symptoms were identified for deaf students.

2. The results $(n=2)$ of Deaf people with Wardenburg syndrome type II were extracted.

3. Deaf students with Wardenburg syndrome type II were divided into classes according to the variable degree of hearing impairment (simple, moderate and severe).

4. Symptoms of the syndrome were depicted on (2) Deaf people with Wardenburg syndrome type II.

5. The performance section of the (WISC-4) scale was applied to the deaf.

\section{The Study Results}

Study questions will be answered according to their sequence:

Results for the first question: What is the concept of deaf people with Wardenburg syndrome type II on a Jordanian sample?

The researcher defined the concept of deaf people with Wardenburg syndrome type II through a set of symptoms that have been studied on deaf in terms of:

- Sensory hearing loss.

- The presence of a side shift to the inner angle of the eyes (through the calculation formula 
previously mentioned).

- Variation in skin pigmentation.

- Premature graying of hair on the front of the head in white and some on the eyelash or on the eyebrow.

- The eyebrows are bound together (excess eyebrows sticking together in the midline).

- Blue eyes, dark brown, or dark pigmented iris.

- The root of the nose is wide.

- The presence of the rabbit's lip.

And through the following cases we review the following:

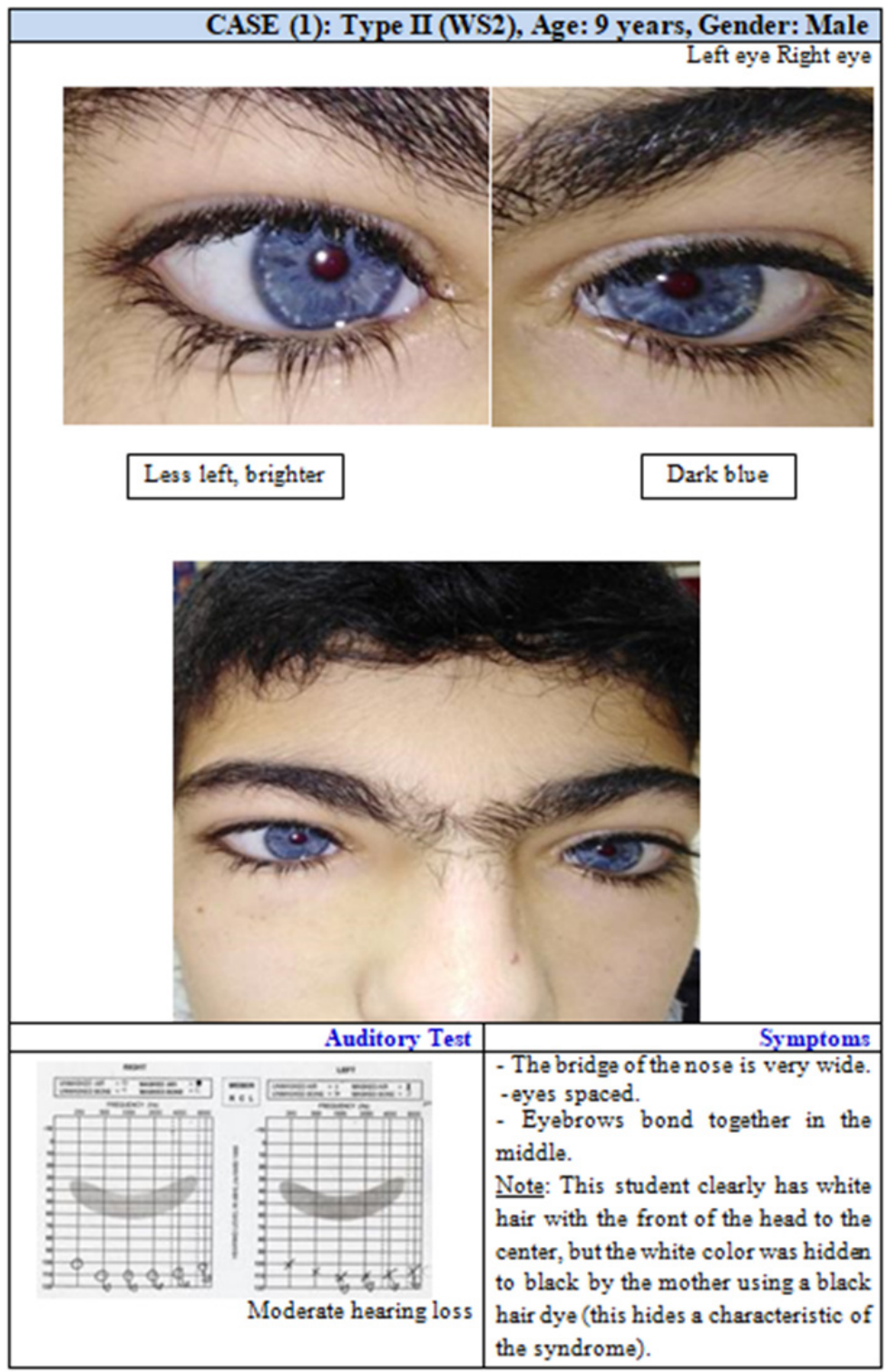




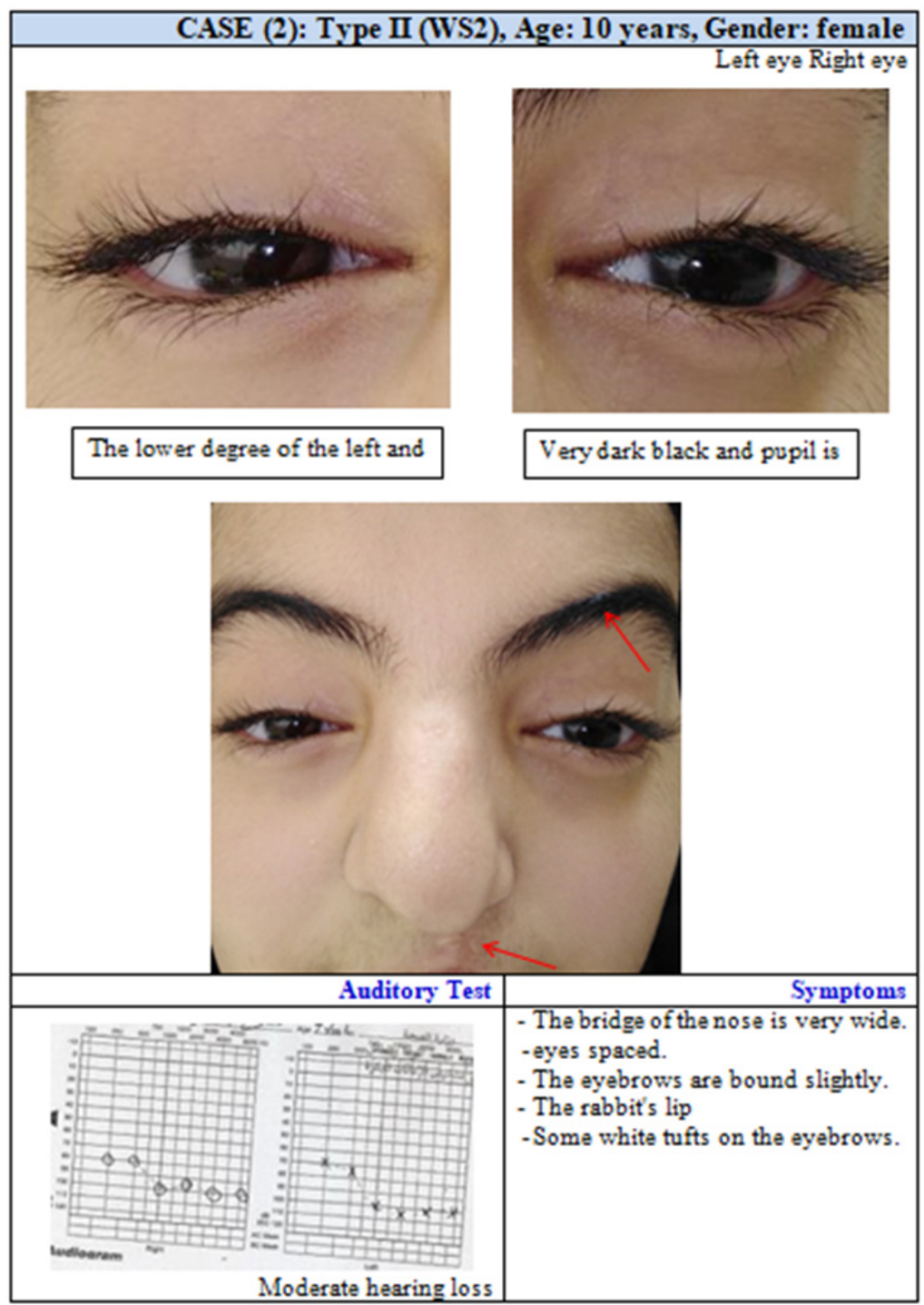

Results related to the second question: What are the characteristics of Deaf people with Wardenburg syndrome type II on a Jordanian sample?

Deaf people with Wardenburg syndrome type II showed some characteristics that deaf their deaf peers from other groups:

First: indicative characteristics:

- Intense focus while communicating in sign language at the hands of the speaker due to the fact that heterogeneity of eyes led to a dispersion of focus in sign language.

- Establishing a space during the indicative communication to clarify the vision of the sign, especially for the deaf with the right eye blue and the left half blue and the other half brown.

Second: optical properties:

Deaf people with Wardenburg syndrome and because of the lack of eye pigmentation, they add some shapes to the image, believing it is correct. For example: 


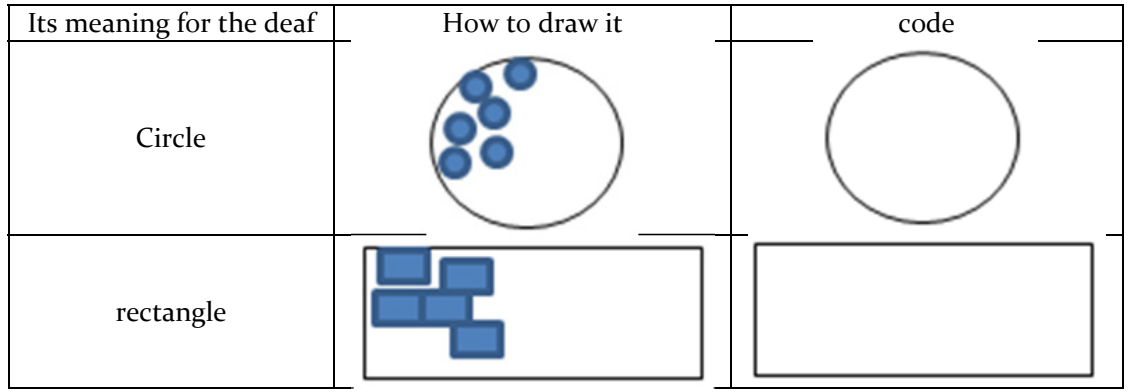

- Deaf people with Wardenburg syndrome type II have a sensitivity to light direction, which may adversely affect the process of visual communication of sign language.

Results for the third question: What is the level of auditory impairment for deaf people with Wardenburg syndrome type II on a Jordanian sample?

Frequencies and percentages were extracted to identify the degree of auditory impairment in Deaf people with Wardenburg syndrome type II, and Table (3) shows that:

Table (3): Distribution of study sample individuals according to the degree of hearing impairment

\begin{tabular}{|c|c|c|}
\hline percentage & Repetition & degree of hearing impairment \\
\hline 100.0 & 2 & moderate \\
\hline 100.0 & 2 & Total \\
\hline
\end{tabular}

\section{Discussion}

Discussion of the results of the first question:

Discussion of the first question: What is the concept of deaf people with Wardenburg syndrome type II on a Jordanian sample?

The results of the study showed that there are symptoms in Deaf people with Wardenberg type II syndrome in terms of:

Sensory hearing loss. The presence of a side shift to the inner corner of the eyes. Premature graying of hair on the front of the head in white and some on the eyelashes or on the eyebrow. The eyebrows are bound together. Blue or dark brown eyes with black and pigment color variations in the iris. The root of the nose is wide and the presence of the rabbit's lip.

The results of this study were in agreement with each of the studies (Jalilian et al, 2018), with the presence of variation in the prominent features of Wardenburg syndrome of the second type. The presence (variation) of iris is the most common $(67 \%$ and $45 \%)$, respectively. The results of this study were consistent with each of the studies (Liu, Newton, Read, 1995) with the occurrence of only one pigmentation disorder. The results of this study were also consistent with each of the studies (Karan, Archana, 2015) with a prominent white introduction to hair. Double blue iris. White pigmentation on the arm. The results of this study also agreed with a study (Kritika, Indu, Gaurav, 2019) with blue eyes. And the presence of bonding with the eyebrows and the presence of a wide distance between the corners of the eye, as well as the front of the hair were white grayish-colored, and pigmented spots in the hands and feet and the nose root was wide. The results of this study were also consistent with the study (Luma, Mohamed, Majd, 2018) of an eye color variation.

The results of this study differed with all studies by the absence of cases of dark brownish black color with both eyes, as well as the presence of the rabbit lip.

The researcher explains : Because many researchers have not mastered the sign language this has led to the lack of discovery of cases within the category of hearing disability because it is a heterogeneous group, and the subject of syndromes accompanying the hearing disability is limited to 
the medical field, this led to a lack of knowledge of the educational and psychological characteristics and mental ability of that group as well as the emergence of Pigmentation with new colors such as black which did not appear clearly in studies, this led to tracing new characteristics of this syndrome.

Discussion of the second question: What are the characteristics of Deaf people with Wardenburg syndrome type II on a Jordanian sample?

The results of the study showed that there is a severe focus during communication in the sign language at the hands of the speaker due to the fact that the color contrast of the eyes led to a dispersion of the focus in the sign language. As well as setting a distance during the sign communication to clarify the vision of the signal, especially for the deaf people with the right eye blue and the left half of it is blue and the other half is brown. They also have a sense of the direction of light, which may adversely affect the process of visual communication of sign language.

The results of this study did not agree or differ with previous studies due to the recent research on this syndrome, especially with regard to communication in sign language and showing some visual sensitivity while communicating with them.

The results of this study were in agreement with the Abu Durei study (2017) that the Jordanian image from the Wexler-4 scale was able to identify some visual problems for this syndrome through performance tests represented by the coding test.

The researcher explains: that the sign language depends on the use of hands in the communication process, which are rapid, accurate and interlinked signal movements between them and therefore if there are visual problems for the deaf it will affect the process of receiving the language being a visual language and may happen here either not seeing some fingers or adding some visual effect On the sign language to distort the language.

Discussion of the third question : What is the level of hearing impairment for Deaf people with Wardenburg syndrome type II on a Jordanian sample?

The results of the study showed that the average degree of hearing impairment is the characteristic of this group.

The results of this study were consistent with the study of (Jian, Frederic, Yong, Paul, Kris, Ingeborg $\mathrm{M}, 2015)$ that auditory loss was $(71 \%)$ and was significantly associated with Wardenburg syndrome. Also, the results of this study were consistent with the study of each From (Jalilian et al, 2018) with sensory hearing impairment. The results of this study were also consistent with each of the study (Liu, Newton, Read, 1995) with the presence of bilateral sensorineural hearing loss. Also, the results agreed with the study (Karan, Archana, 2015) with the presence of auditory sensory impairment. The results of this study were consistent with (Luma, Mohamed, Majd, 2018) study regarding the presence of congenital sensory hearing loss.

The researcher explains: Because of the auditory sensory loss of deaf people with Wardenburg syndrome type II and associated with the inner ear, which is the feature of this syndrome, all of its categories have a hearing loss of medium degree, and this indicates the use of sign language instead of lip language due to the proportion of auditory loss for that category.

\section{Recommendations}

Based on the findings: Educational recommendations:

1. Inclusion of this syndrome as an important part of the categories of hearing impairment.

2. Training for deaf workers on the characteristics of the Wardenburg type II syndrome.

3. Training workers to know the visual problems resulting from eye pigmentation, which may add shapes that are not present.

Research Recommendations:

1. Carrying out more studies and research related to Wardenburg syndrome of the second type for the hearing impaired.

2. Carrying out studies related to mental disorders in the Deaf with Type II syndrome.

3. Carry out studies related to visual perception in Deaf people with Type II syndrome. 
4. Carrying out studies related to the level of intelligence of the deaf people with Type II syndrome.

Conclusion : The lack of researchers specialized in sign language at the level of translation of the deaf language makes it difficult to know and discover other cases accompanying hearing disability (such as double disability), and therefore there are many other cases that must be known in order to identify areas and levels within the category of hearing disability, which helps us to enrich this field Hence the secret in this scientific research lies in knowing the characteristics and rules of the sign language for the deaf in the researcher due to the ease of identification and access to other cases that do not appear with the normal measuring tools for that category.

\section{References}

Abu Drei, S.(2017).Standardization A Jordanian Version of The Wechsler Intelligence Scale for Children -Fourth Edition Adapted for Deaf Via Sign Language From( 6-16,11) years. Phd dissertation.Amman, Jordan: The Word Islamic Science and Education University.

Adameyko, I, Lallemend, F, Aquino, JB, et al(2009). Schwann cell precursors from nerve innervation are a cellular origin of melanocytes in skin. Cell.; 139( 2):366- 379.

Dourmishev LA, Elston DM D(2013) . ermatologic Manifestations of Waardenburg syndrome clinical presentation. http://emedicine. Medscape .com/article/ 1113314-clinical\#ao217.

Dourmishev AL, Dourmishev LA, Schwartz RA,(1999).Waardenburg syndrome.Int J Dermatol, ;38(9): 656-63.

Egbalian .F.(2008) .Waardenburg Shah Syndrome; A Case Report and Review of the Literature. Iranian Journal of Pediatrics 18(1), Pp:71-74.

Farrer LA, Grundfast KM, Amos J, Arnos KS, Asher JH, Beighton P, Diehl SR, Fex J, Foy C, Friedman TB, et al. (1992). Waardenburg syndrome (WS) type I is caused by defects at multiple loci, one of which is near ALPP on chromosome 2: first report of the WS consortium.Am J Hum Genet 50:902-13.

Gorlin RJ, et al(2010). Syndromes of the Head and Neck 5 .th ed .New York, NY: Oxford University Press:1369

Hart Joseph , Kalpana Miriyala,.(2017). Neural tube defects in Waardenburg syndrome: A case report and review of the literature. American Journal of Medical Genetics Part AVolume 173, Issue 9.

Harlor, A. D., Jr., \& Bower, C. (2009). Hearing assessment in infants and children: Recommendations beyond neonatal screening. Pediatrics, 124(4), 1252-1263.

Hager, T; Walter, H-S; Seitz, B; KÃ đ̊smann-Kellner, B.(2010). Visual diagnosis: Waardenburg syndrome. in Der Ophthalmologe 107 (7) : 66o-2.

Jalilian Nazanin, Mohammad Amin Tabatabaiefar, Mahboubeh Yazdanpanah4, Elham Darabi, Tayyeb Bahrami , Ali Zekri , Mohammad Reza Noori-Daloii.(2018). A Comprehensive Genetic and Clinical Evaluation of Waardenburg Syndrome Type II in a Set of Iranian Patients. IJMCM Original Article Winter, Vol 7, No 1 .

Jones KL.(2006). Smith's Recognizable Patterns of Human Malformation. 6 th ed . Philadelphia , PA: WB Saunders Compan-79-278.

Jian, S ; Frederic ,A ; Yong ,F ; Paul ,C; Kris ,V, Ingeborg,D .(2015). Hearing loss in Waardenburg syndrome: A systematic review. Article in Clinical Genetics 89(4).

Karan Sharma , Archana Arora(2015). Waardenburg Syndrome: A Case Study of Two Patients. Indian J Otolaryngol Head Neck Surg.; 67(3):324-328

Kritika Katoch, Indu Dhiman, Gaurav SharmaA (2019). Case Study of Two Siblings of Waardenburg Syndrome.DJO;29:111-113

Klein, D (1983). Klein Historical background and evidence for dominant inheritance of the Klein-Waardenburg syndrome (type III) Am. J. Med. Genet., 14 , pp. 231-239.

Luma Haj Kassem, Mohamed Fares Ahmado, Majd Sheikh Alganameh(2018) .A rare case of seven siblings with Waardenburg syndrome: a case report .Journal of Medical Case Reports volume 12, Article number: 192.

Liu XZ, Newton VE, Read AP(1995). Waardenburg syndrome type II: phenotypic findings and diagnostic criteria. Am J Med Genet.;55:95-100

Melville, C. and Cameron, J. (2003). Autism, Seminars in the psychiatry of learning disabilities. Gaskell publication, 124.

Milunsky JM, Maher TA, Ito M, Milunsky A(2007). The value of MLPA in Waardenburg syndrome. Genet Test.;11:179-82.

National Institute on Deafness and Other Communication Disorders (NIDCD). (2007).Waardenburg Syndrome. 16 Apr. 
Nayak, Chetan S; Isaacson, Glenn.(2003). Worldwide distribution of Waardenburg syndrome. The Annals of otology, rhinology, and laryngology $112(9 \mathrm{Pt} 1): 817-20 \cdot 70$.

Nitin Vichareand, N. Bhargava (2013). Waardenburg syndrome: A rare case with bilateral congenital cataract: An unusual entity. Med J Armed Forces India.; 69(2): 172-174.

Orphanet.(2018).Waardenburg syndrome. www.orpha.net/consor/cgi-bin /Disease. Oiso N, Fukai K, Kawada A, Suzuki T. Piebaldism. J Dermatol. 2013;40(5):330-335.

Pasteris NG, et al.(1992). A chromosome deletion 2935-36 spanning loci $\mathrm{HuP}_{2}$ and $\mathrm{COL}_{4} \mathrm{~A}_{3}$ results in Waardenburg syndrome type III (Klein-Waardenburg syndrome) .Am J Hum Genet .51 A224.

Pantke, M.M. Cohen Jr(1971).The Waardenburg syndrome Birth Defects.,7, pp.147-152.

Pingault, V., Ente, D., Dastot-Le Moal, F., Goossens, M., Marlin, S., \& Bondurand, N. (2010). Review and update of mutations causing Waardenburg syndrome. Human Mutation, 31(4), 391-406.

Shah, K.N. Shah (1981). White forelock, pigmentary disorder of irises, and long segment Hirschsprung disease: possible variant of Waardenburg syndrome J. Pediatr. , 99, pp. 432-434.

Shields CL, Nickerson SJ, Al-Dahmash S, Shields JA(2013). Waardenburg syndrome: iris and choroidal hypopigmentation: findings on anterior and posterior segment imaging. JAMA Ophthalmol.131:1167-73.

Steel, K. P. and Barkway, C. (1989). Another role for melanocytes: their importance for normal stria vascularis development in the mammalian inner ear. Development, 107, 453-63.

Tagra S, Talwar AK, Walia RL, (2006).Waardenburg syndrome.Indian J Dermatol Venereoleprol.;72(4):326.

Toriello, H. V., Reardon, W. and Gorlin, R. J. (2004). Hereditary hearing loss and its syndromes. Oxford University Press.

USNLM ,U.S. National Library of Medicine (2006). Waardenburg Syndrome.

Waardenburg PJ.(1951). A new syndrome combining developmental anomalies of the eyelids, eyebrows and nose root with pigmentary defects of the iris and head hair and with congenital deafiess. Am J Hum Genet;3:195253. 